reusable-part condenser [4], and results obtained by others using a non-reusable-part condenser and mass spectrometry (MS) [7].

The data mentioned previously demonstrate that the cleaning procedure with Milton does not alter the metabolic profiles of $\mathrm{EBC}$, and that NMR spectroscopy is suitable for investigating EBC samples.

By using an Anacon condenser (Biostec, Valencia, Spain), different results have been reported [7]. Collection devices are an important source of variability of EBC biomarkers [2, 3, 8]. The principal variability factors include cooling temperature [9] and condenser materials [2]. For example, a warm-up during condensation is observed when RTube or Anacon are used (condensers using a disposable collection kit), whereas EcoScreen cools down slightly during the procedure [8,9]. Such differences affect biomarker concentrations $[2,9]$. More importantly, there was no correlation between biomarkers measured in EBC collected with EcoScreen and Anacon condensers [9], and there was only an $\sim 60 \%$ correlation between biomarkers measured in EBC collected with RTube and Anacon condensers; although both used a disposable collection kit [9]. However, as no NMR details were found (i.e. the operating magnetic field, the number of acquisitions, the possible use of a cryoprobe, and the detection limit), it is difficult to conclude that NMR spectroscopy cannot be used to analyse EBC samples because it does not have the sensitivity required to observe the endogenous metabolites in the EBC [7]. With our spectrometer set-up, we have estimated a detection limit that is notably low for NMR-based metabolomics [10].

In conclusion, our cleaning procedure of EBC collection set-up does not generate artificial signals in the metabolic profile of EBC. Furthermore, NMR-based metabolomics are suitable for identifying specific EBC metabolites and are potentially useful for characterising the metabolic fingerprints of patients with respiratory diseases. Although a head-to-head comparison of different condensers is required, the combination of different reference analytical techniques, including NMR and MS, might consolidate "breathomics" as a new noninvasive approach to the assessment of patients with respiratory disease with important diagnostic and therapeutic implications.

A. Motta*, D. Paris*, D. Melck*, G. de Laurentiis", M. Maniscalco\#, M. Sofia" and P. Montuschi ${ }^{*}$

*Institute of Biomolecular Chemistry, National Research Council, Pozzuoli, "Dept of Respiratory Medicine, A.O. Monaldi,
University of Naples "Federico II", Naples, and "Dept of Pharmacology, Faculty of Medicine, Catholic University of the Sacred Heart, Rome, Italy.

Correspondence: P. Montuschi, Dept of Pharmacology, Faculty of Medicine, Catholic University of the Sacred Heart, Largo Francesco Vito 1, I-00168 Rome, Italy. E-mail. pmontuschi@rm. unicatt.it

Statement of Interest: None declared.

\section{REFERENCES}

1 Horvath I, Hunt J, Barnes PJ. Exhaled breath condensate: methodological recommendations and unresolved questions. Eur Respir J 2005; 26: 523-548.

2 Rosias P, Robroeks C, Kester A, et al. Biomarker reproducibility in exhaled breath condensate collected with different condensers. Eur Respir J 2008; 31: 934-942.

3 Koczulla R, Dragonieri S, Schot R, et al. Comparison of exhaled breath condensate $\mathrm{pH}$ using two commercially available devices in healthy controls, asthma and COPD patients. Respir Res 2009; 10: 78.

4 de Laurentiis G, Paris D, Melck D, et al. Metabonomic analysis of exhaled breath condensate in adults by nuclear magnetic resonance spectroscopy. Eur Respir J 2008; 32: 1175-1183.

5 Carraro S, Rezzi S, Reniero F, et al. Metabolomics applied to exhaled breath condensate in childhood asthma. Am J Respir Crit Care Med 2008; 175: 986-990.

6 Montuschi P, Paris D, Melck D, et al. Metabolomic analysis by nuclear magnetic resonance spectroscopy of exhaled breath condensate in patients with cystic fibrosis. Eur Respir J 2009; 34: $63 \mathrm{~s}$.

7 Izquierdo-García JL, Peces-Barba G, Heili S, et al. Is NMR-based metabolomic analysis of exhaled breath condensate accurate? Eur Respir J 2011; 37: 468-470.

8 Hoffmeyer F, Raulf-Heimsoth M, Harth V, et al. Comparative analysis of selected exhaled breath biomarkers obtained with two different temperature-controlled devices. BMC Pulm Med 2009; 9: 48.

9 Czebe K, Barta I, Antus B, et al. Influence of condensing equipment and temperature on exhaled breath condensate $\mathrm{pH}$, total protein and leukotriene concentrations. Respir Med 2008; 102: 720-725.

10 del Campo G, Berregi I, Caracena R, et al. Quantitative determination of caffeine, formic acid, trigonelline and 5-(hydroxymethyl)furfural in soluble coffees by ${ }^{1} \mathrm{H}-\mathrm{NMR}$ spectrometry. Talanta 2010; 81: 367-371.

\title{
Metabolomic signatures in nuclear magnetic resonance spectra of exhaled breath condensate identify asthma
}

\section{To the Editors:}

Exhaled breath condensate (EBC) holds promise as a noninvasive method of collecting airway-lining fluid, although at an unknown dilution [1]. While metabolomic studies of EBC using nuclear magnetic resonance (NMR) spectroscopy have previously shown promise in asthma diagnosis and subtyping [2,3], a study that was recently published in the European Respiratory Journal that failed to find usable NMR signature in EBC collections from disposable systems [4]. This led the authors to conclude that NMR metabolomics lacks sufficient sensitivity for metabolic fingerprinting of EBC. Interestingly, they were able to obtain high quality results from the same samples with mass spectroscopy, which they recommended for future use. As this is a nascent and 
technically complex field, we present our very different early experiences, which suggest that reproducible, valid and useful NMR metabolomic fingerprinting of EBC is indeed possible. Specifically, we found that the presence or absence of a trident peak at 7 ppm during NMR spectroscopy reliably distinguished between EBC samples collected from normal and asthmatic subjects, respectively. This peak probably represents ammonium ion, loss of which in asthma is consistent with reduced expression of glutaminase, an enzyme that converts glutamine to glutamate and ammonia [5].

Institutional committees approved all research protocols. The subjects included both adults ( $>21 \mathrm{yrs})$ and children (6-15 yrs). Adult asthmatic subjects $(n=7)$ were physician diagnosed, meeting American Thoracic Society/European Respiratory Society criteria [6], and were nonsmokers. Control normal adults were healthy nonsmoking volunteers $(n=10)$. The children were part of an ongoing prospective asthma cohort for which multidimensional genotype and phenotype data is being collected $(n=58)$. The goal of this cohort is asthma subphenotype discovery, and no controls exist within the cohort. Two EBC samples from nonasthmatic children with no personal or family history of respiratory disease were additionally collected. EBC samples were collected using a completely disposable collection system (Rtube; Respiratory Research, Austin, TX, USA), over a 10-min period by standard manufacturer protocol [7].

${ }^{1} \mathrm{H}-\mathrm{NMR}$ spectra were obtained on a $600 \mathrm{MHz}$ NMR Spectrometer (Bruker, Germany) equipped with a triple resonance inverse (TCI) probe. Water suppression was done using a modified nuclear Overhauser effect spectroscopy (NOESY) sequence. ${ }^{1} \mathrm{H}-\mathrm{NMR}$ spectra of water from RTube collection tubes (disposable tubes of plastic) was first obtained to define any contaminants originating from the collection tubes themselves. This and representative spectra from asthmatic and control subjects are shown in figure 1. It was seen that: 1) EBC collections from human samples in disposable tubes have characteristic signatures during NMR spectroscopy; 2) these are different from experimental control spectra obtained from the tube itself; and 3) there is a trident peak near 7 ppm that is different between asthmatic subjects and controls. Only one (14\%) out of seven adult asthmatic subjects had the peak, which was in sharp contrast to eight $(80 \%)$ out of 10 of control subjects. This was statistically significant (peak present/peak absent in asthmatic subjects versus controls; $1 / 6$ versus $8 / 2$, OR 24; $\mathrm{p}=0.01$ ). Regarding children, we had predominantly asthmatic subjects and only two control subjects, but the results were still statistically significant (peak present/peak absent in asthmatic subjects versus controls; $4 / 54$ versus $2 / 0 ; \mathrm{p}=0.0085$ ) and, more importantly, consistent with the findings that, in adults, $\sim 90 \%$ of asthmatic subjects did not show the peak, while normal subjects did. No major age-related differences were identified in the EBC spectra and further analysis in a machine learning system for subphenotype discovery is in progress. Biological significance of the difference at 7 ppm was further ascertained.

The trident peak at $7 \mathrm{ppm}$ was found to be due to the ammonium ion, and has been confirmed by the addition of ammonium chloride to the samples. In healthy subjects, glutaminasemediated synthesis of ammonia maintains airway $\mathrm{pH}$ by buffering free hydrogen ions $\left(\mathrm{NH}_{3}+\mathrm{H}^{+}=\mathrm{NH}_{4}{ }^{+}\right)$. It has been previously shown by HUNT et al. [5] that in patients with acute

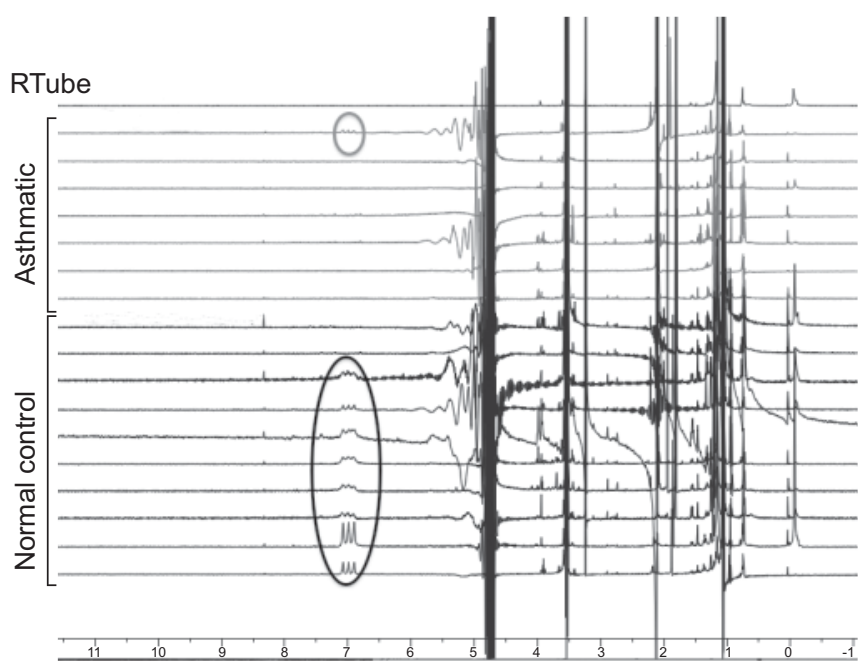

FIGURE 1. Absence of a trident peak at $7 \mathrm{ppm}$ in nuclear magnetic resonance (NMR) spectra of exhaled breath condensate (EBC) is associated with asthma. ${ }^{1} \mathrm{H}$ NMR spectra of EBCs collected from 10 normal and seven asthmatic adults are shown. Experimental control (top trace) from the collection system (RTube) is clearly different from the EBC spectra. The oval box highlights a trident peak at 7 ppm seen in most normal subjects (lower 10 traces) but is missing in most asthmatic subjects (middle seven traces). Peak present/peak absent in asthmatic subjects versus controls ( $1 / 6$ versus $8 / 2$, OR 24; $p=0.01$ )

asthma exacerbations, airways are more acidic and ammonia content is lower in comparison with normal subjects [5]. Whether there is a loss of acid neutralisation capacity due to reduced glutaminase activity in chronic asthma is less clear. HuNT et al. [5] found in vitro evidence of acid-dependent expression of glutaminase in human lung adenocarcinoma cell lines, which was suppressed by inflammatory cytokines. As serum glutaminase activity has been shown to reflect tissue glutaminase activity in some models [8], we determined the glutaminase activity in sera of asthmatic subjects and controls. Using an ELISA-based assay (USCNK Life Sciences, Wuhan, China), we found that glutaminase levels were significantly higher in normal controls compared with asthmatic subjects (fig. 2).

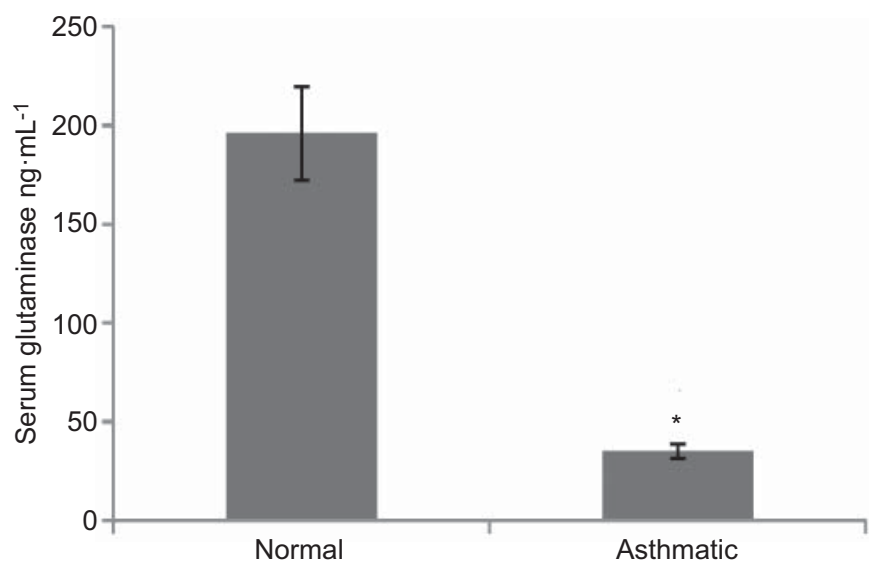

FIGURE 2. Serum glutaminase is reduced in asthma. Serum glutaminase levels, measured by ELISA, are shown for normal and asthmatic subjects. *: $p<0.05$. 
In summary, we show for the first time that the absence of a trident peak at $7 \mathrm{ppm}$ in NMR spectra of $\mathrm{EBC}$, related to ammonium, is associated with asthma. The loss of ammonium reflects reduced ammonia synthesis due to downregulation of glutaminase, leading to impaired acid neutralisation. Surprisingly, despite a very strong association in our study, this signature was not noted in the seminal study by CARRARO et al. [2], who first described the use of NMR spectroscopy of EBC in asthma. Only spectra between 1 and 4 ppm were shown in their study. One possibility for the observed differences is that CARRARO et al. [2] used a reusable collection system. It is possible, as suggested by IZQUIERDO-GARCíA et al. [4], that the use of disinfectants in reusable collection systems may create artefacts, cleaning of which may in turn obscure some parts of the spectra. As we use a completely disposable tube for EBC collection, with only a chilled external metal sleeve being reused, there is no possibility of such contamination in our data. It was additionally confirmed that EBC spectra including the 7 ppm peaks were distinct from deuterated water condensate collected from identical tubes. Furthermore, independent previous reports of ammonia and ammonium being reduced in asthma corroborate our findings $[5,9]$. The small number of controls in our study, particularly of children, limits our study and further studies are needed to establish the usefulness of our finding. Also, it appears likely that the ammonium signature is enhanced by acidic load on the airway, which may vary across geographical regions. This study was conducted in Delhi, India, where ambient air contains high sulphuric and nitric oxides. Applicability of our finding to less polluted regions needs to be tested. However, despite this, our data hopefully removes the need for any further debate regarding whether valid and useful NMR spectra can be obtained for EBC [4]. This nascent field holds much potential for translation, and concerted efforts of many investigators are needed.

\section{A. Sinha*, V. Krishnan*, T. Sethi*, S. Roy", B. Ghosh*, R. Lodha", S. Kabra" and A. Agrawal*}

${ }^{*}$ Center of Excellence, Translational Research in Asthma and Lung Disease, Council of Scientific and Industrial Research (CSIR) Institute of Genomics and Integrative Biology, "Dept of Paediatrics, All India Institute of Medical Sciences, Delhi, and "Structural Biology and Bioinformatics, CSIR-Indian Institute of Chemical Biology, Kolkata, India.
Correspondence: A. Agrawal, Institute of Genomics and Integrative Biology, Mall Road, Delhi 110007, India. E-mail: a.agrawal@igib.in

Support Statement: The study was funded by the Centre for Excellence Project MLP 5501 of Council of Scientific and Industrial Research (CSIR), Government of India. A. Agrawal acknowledges the support of the Lady Tata Memorial Trust.

Statement of Interest: None declared.

Acknowledgements: The authors acknowledge research funding support from CSIR project MLP5501. They thank members of the Dept of Paediatrics, All India Institute of Medical Sciences, Delhi, especially M.B. Meenakshi, A.P. Anibha, B.J. Bipin and H.P. Hrishikesh, for collection of patient samples and to the Dept of Structural Biology and Bioinformatics at IICB, Kolkata, India, with a special thanks to E.P. Padmanabhan for help in performing NMR spectroscopy.

\section{REFERENCES}

1 Horváth I, Hunt J, Barnes PJ, et al. ATS/ERS Task Force on Exhaled Breath Condensate. Eur Respir J 2005; 26: 523-548.

2 Carraro S, Rezzi S, Reniero F, et al. Metabolomics applied to exhaled breath condensate in childhood asthma. Am J Respir Crit Care Med 2007; 175: 986-990.

3 De Laurentiis G, Paris D, Melck D. Metabonomic analysis of exhaled breath condensate in adults by nuclear magnetic resonance spectroscopy. Eur Respir J 2008; 32: 1175-1183.

4 Izquierdo-García JL, Peces-Barba G, Heili S, et al. Is NMR-based metabolomic analysis of exhaled breath condensate accurate? Eur Respir J 2011; 37: 468-470.

5 Hunt JF, Erwin E, Palmer L, et al. Expression and activity of pHregulatory glutaminase in human airway epithelium. Am J Respir Crit Care Med 2002; 165: 101-107.

6 Reddel HK, Taylor DR, Bateman ED, et al. An Official American Thoracic Society/European Respiratory Society Statement: Asthma Control and Exacerbations. Am J Respir Crit Care Med 2009; 180: 59-99.

7 Alix $\mathrm{O}$, Brown $\mathrm{P}, \mathrm{Ngam}$ trakulpanit L, et al. Normative data for $\mathrm{pH}$ of exhaled breath condensate. Chest 2006; 129: 426-430.

8 Petrun NM, Migal LA, Drannik GN. Activity of glutamine deaminating enzymes in the kidney, liver and serum of dogs with renal failure and under normal conditions. Vopr Med Khim 1977; 23: 485-489.

9 MacGregor G, Ellis S, Andrews J, et al. Breath condensate ammonia is lower in children with chronic asthma. Eur Respir J 2005; 26: 271-276.

\section{Widespread use of serological tests for tuberculosis: data from 22 high-burden countries}

\section{To the Editors:}

There is great excitement over the introduction of new tuberculosis (TB) diagnostics [1]. Since 2007, several TB diagnostics and approaches have been endorsed by the World Health Organization (WHO) [2],with Xpert MTB/RIF (Cepheid, Sunnyvale, CA, USA) being the most recent [3]. Amidst this excitement, there is growing concern surrounding the use of inappropriate and suboptimal TB diagnostics [4,5].

Currently available commercial serological (antibody detection) tests for TB are inaccurate and highly inconsistent [6-8]. The International Standards for TB Care explicitly discourage their use [9]. Even so, serological tests are known to be widely used in 